\title{
Drug Resistance to Anti-Tuberculosis Drugs: A Cross-Sectional Study From Makkah, Saudi Arabia
}

Ahmad M. Al-Hayani ${ }^{1}$, Shady A. Kamel ${ }^{1}$, Sami S. Almudarra ${ }^{1}$, Majed Alhayani ${ }^{2}$, Ahmed Abu-Zaid ${ }^{3}$

1. Saudi Field Epidemiology Training Program, Ministry of Health, Riyadh, SAU 2. College of Medicine, King Abdulaziz University, Jeddah, SAU 3. College of Graduate Health Sciences, The University of Tennessee Health Science Center, Memphis, USA

Corresponding author: Ahmad M. Al-Hayani, ahmadmh.amh@gmail.com

\section{Abstract}

\section{Aim}

The aim of this study was to investigate the patterns and determinants of drug resistance to tuberculosis (TB) in a large population from Makkah, Saudi Arabia.

\section{Methods}

A retrospective, cross-sectional cohort study was conducted on all patients with TB who were referred to the National Tuberculosis Prevention Program in Makkah, Saudi Arabia, between January 2016 and September 2020. For each TB case, demographic data were collected in addition to the results of drug susceptibility testing (DST) for anti-TB drugs. The Statistical Package for Social Sciences (SPSS) software for Windows, version 23 (IBM Corporation, Armonk, NY, USA), was used for the statistical analysis.

\section{Results}

A total of $472 \mathrm{~TB}$-confirmed cases were included in the analysis. The mean \pm standard deviation of the age was $38.5 \pm 17.7$ years. The vast majority of patients were male $(62.7 \%)$ and had pulmonary TB (91.7\%). Only a small proportion of the patients with TB had diabetes mellitus (8.5\%). Overall, the prevalence of monodrugresistant TB ranged from $2.1 \%$ to $3.4 \%$. Specifically, the prevalence of monodrug-resistant TB to isoniazid and streptomycin was ranked first and was equal to 3.4\%. Pyrazinamide had the lowest prevalence of monodrug-resistant TB (2.1\%). The prevalence of polydrug-resistant TB (PDR-TB) and multidrug-resistant TB (MDR-TB) was $1.5 \%$. In the univariate analysis, sex (male) was the only sociodemographic factor that significantly correlated with a higher prevalence of MDR-TB.

\section{Conclusions}

This is the second study from Makkah to analyze the prevalence and associated risk factors of MDR-TB among patients from Makkah. Our data demonstrated that the prevalence of monodrug-resistant TB and MDR-TB was low (2.1\%-3.4\% and 1.5\%, respectively). Diabetes mellitus was not a substantial factor

Review began $07 / 29 / 2021$ Review ended 07/31/2021 Published 08/10/2021

(๑) Copyright 2021

Al-Hayani et al. This is an open access article distributed under the terms of the Creative Commons Attribution License CC-BY 4.0., which permits unrestricted use, distribution, and reproduction in any medium, provided the original author and source are credited. correlated with a higher occurrence of MDR-TB. Additional epidemiologic studies are required to validate our results.

Categories: Infectious Disease, Public Health, Epidemiology/Public Health

Keywords: tuberculosis, multidrug-resistant tuberculosis, polydrug-resistant tuberculosis, makkah, saudi arabia, isoniazid, rifampicin

\section{Introduction}

Tuberculosis (TB) continues to be a chief healthcare predicament worldwide and a prominent cause of mortality from a solitary infectious disease agent [1]. More than 10 million new cases of TB and 1.5 million mortalities were documented in 2018 [2]. By the end of 2035, the World Health Organization (WHO) aspires to accomplish a dramatic decline in the frequency of TB and its mortality by $90 \%$ and $95 \%$, respectively [3].

Although TB can be successfully cured in the vast majority of patients, some individuals develop resistance to first-line anti-TB therapy, comprising isoniazid, rifampicin, pyrazinamide, ethambutol, and streptomycin [4]. Polydrug-resistant TB (PDR-TB) occurs when a patient develops resistance to two or more first-line antiTB agents other than isoniazid and rifampicin [5]. On the other hand, multidrug-resistant TB (MDR-TB) occurs when a patient develops resistance to both isoniazid and rifampicin with or without other first-line agents [6]. Approximately half a million TB cases are diagnosed with MDR-TB [2]. Unfortunately, MDR-TB is associated with costly treatment expenses, lower rates of treatment success, and higher burdens of morbidity and mortality [7-9].

TB remains a major public health issue in Saudi Arabia. According to the most recent statistics, the yearly TB incidence is 10 per 100,000 individuals [2]. A large national study from Saudi Arabia demonstrated that MDR-TB rates among new and previously treated cases were $1.8 \%$ and $15.9 \%$, respectively [10]. Other national registry data from the Ministry of Health stated that the prevalence rate of MDR-TB was 4.4\% [11]. 
The occurrence rate of MDR-TB varies according to the region/city of the report [12], and these geographic differences need to be recognized. Makkah, a city in the Western province of Saudi Arabia, serves as the principal hub for two mass religious gatherings, namely the Hajj and Umrah pilgrimages. While Haji pilgrimage takes place annually by the end of the Hijri calendar, Umrah pilgrimage takes place throughout the year. These two pilgrimages attract millions of individuals worldwide, including Asian and African countries with a high TB burden [1]. Thus, it is critical to explore the prevalence and patterns of MDR-TB in this vulnerable city. So far, only one study has aimed to explore the prevalence and determinants of MDRTB in Makkah [13]. This study was conducted at the Al-Noor Specialist Hospital and evaluated the records of only 158 patients with TB-confirmed cases. Hence, additional large-sized data are warranted to provide more comprehensive and high-quality evidence.

This study aimed to investigate the patterns and determinants of drug resistance to TB among a large sample from Makkah, Saudi Arabia.

\section{Materials And Methods}

The study protocol was approved by the Institutional Review Board of King Abdulaziz City for Science and Technology, Riyadh, Saudi Arabia. A retrospective, cross-sectional cohort study was conducted on all TBconfirmed patients, irrespective of age, who were referred to the National Tuberculosis and Prevention Program in Makkah, Saudi Arabia, between January 2016 and September 2020.

The TB diagnosis was confirmed by various methods, including chest radiography, sputum culture, microscopy, or molecular assays. TB resistance was established using drug susceptibility testing (DST). To isolate and identify the mycobacterium agent, Ziehl-Neelsen (ZN) staining was performed to examine acidfast bacilli. Culture and DST were performed using the mycobacteria growth indicator tube (MGIT) medium and BACTEC MGIT 960 instrument (Becton, Dickinson and Company, Maryland, United States of America), as reported previously [14]. The final drug concentrations utilized for DST included $5 \mu \mathrm{g} / \mathrm{ml}$ for ethambutol, $0.1 \mu \mathrm{g} / \mathrm{mL}$ for streptomycin, $1 \mu \mathrm{g} / \mathrm{mL}$ for rifampicin, $0.1 \mu \mathrm{g} / \mathrm{mL}$ for isoniazid, and $25 \mu \mathrm{g} / \mathrm{mL}$ for pyrazinamide. For selected samples, the GenXpert Mycobacterium tuberculosis complex/resistance to rifampicin (MTB/RIF) assay (Cepheid, California, United States of America) was used to screen for the Mycobacterium tuberculosis complex and resistance to rifampicin.

Demographic data were collected for each TB case (i.e., age, sex, nationality, disease site, diabetes mellitus, renal failure, acquired immunodeficiency syndrome, immunosuppression, cancer, and Bacillus CalmetteGuérin vaccination) in addition to the results of the DST for TB. DST results focused on identifying the rates of monodrug-resistant TB (resistance to one anti-TB drug), PDR-TB (resistance to two or more anti-TB drugs other than isoniazid and rifampicin), and MDR-TB (resistance to at least both isoniazid and rifampicin with or without resistance to other drugs).

The Statistical Package for Social Sciences (SPSS) software for Windows, version 23, was used for statistical analysis (IBM Corporation, Armonk, NY, USA). Descriptive data were presented as numbers and percentages or means and standard deviations, as appropriate. The Chi-squared test was used to examine correlations between select patient demographics and the occurrence of MDR-TB.

\section{Results}

A total of 472 records of TB-confirmed cases were included in the analysis. Table 1 summarizes the sociodemographic characteristics of the patients. The mean \pm standard deviation of age was $38.5 \pm 17.7$ years (range: 2-101 years). The prevalence of TB was equally divided between Saudi and non-Saudi nationals (50.4\% and $49.6 \%$, respectively). Most patients were male (62.7\%) and had pulmonary TB (91.7\%). Only a small proportion of TB patients had diabetes mellitus (8.5\%), renal failure (23\%), acquired immunodeficiency syndrome (3\%), immunosuppression (1.9\%), and cancer (1.5\%). Equally, $5.7 \%$ of patients had a previous history of TB and received the Bacillus Calmette-Guérin vaccine. 


\section{Cureus}

\begin{tabular}{|c|c|}
\hline Sociodemographic & $n(\%)$ \\
\hline \multicolumn{2}{|l|}{ Nationality } \\
\hline Saudi & $238(50.4)$ \\
\hline Non-Saudi & $234(49.6)$ \\
\hline \multicolumn{2}{|l|}{ Sex } \\
\hline Male & $296(62.7)$ \\
\hline Female & $176(37.7)$ \\
\hline \multicolumn{2}{|l|}{ Type } \\
\hline Pulmonary & $433(91.7)$ \\
\hline Extra-pulmonary & $36(7.6)$ \\
\hline Both & $3(0.6)$ \\
\hline \multicolumn{2}{|l|}{ Diabetes mellitus } \\
\hline Yes & $40(8.5)$ \\
\hline No & $432(91.5)$ \\
\hline \multicolumn{2}{|l|}{ Renal failure } \\
\hline Yes & $11(2.3)$ \\
\hline No & $461(97.7)$ \\
\hline \multicolumn{2}{|c|}{ Acquired immunodeficiency syndrome } \\
\hline Yes & $14(3)$ \\
\hline No & $458(97)$ \\
\hline \multicolumn{2}{|l|}{ Immunosuppression } \\
\hline Yes & $9(1.9)$ \\
\hline No & $463(98.1)$ \\
\hline \multicolumn{2}{|l|}{ Cancer } \\
\hline Yes & $7(1.5)$ \\
\hline No & $465(98.5)$ \\
\hline \multicolumn{2}{|c|}{ Bacillus Calmette-Guérin vaccine } \\
\hline Yes & $27(5.7)$ \\
\hline No & $445(94.3)$ \\
\hline \multicolumn{2}{|c|}{ Previous history of tuberculosis } \\
\hline Yes & $27(5.7)$ \\
\hline No & $445(94.3)$ \\
\hline
\end{tabular}

TABLE 1: The sociodemographics of patients.

Table 2 outlines the patterns of drug resistance to anti-TB drugs. Overall, the prevalence of monodrugresistant TB ranged from $2.1 \%$ to $3.4 \%$. Specifically, the prevalence of monodrug-resistant TB to isoniazid and streptomycin was ranked first and was equal to $3.4 \%$. Pyrazinamide had the lowest prevalence of monodrug-resistant TB (2.1\%). The prevalence of PDR-TB and MDR-TB was $1.5 \%$. 


\section{Cureus}

\begin{tabular}{|l|l|}
\hline Type of tuberculosis resistance & $\mathrm{n}(\%)$ \\
\hline Monodrug-resistant tuberculosis & $16(3.4)$ \\
\hline Isoniazid & $10(2.1)$ \\
\hline Pyrazinamide & $12(2.5)$ \\
Ethambutol & $13(2.8)$ \\
Rifampicin & $16(3.4)$ \\
Streptomycin & \\
Polydrug-resistant tuberculosis & $5(1.1)$ \\
Pyrazinamide plus ethambutol & $7(1.5)$ \\
Pyrazinamide plus streptomycin & $7(1.5)$ \\
Ethambutol plus streptomycin & $7(1.5)$ \\
Multidrug-resistant tuberculosis &
\end{tabular}

TABLE 2: Patterns of resistance to anti-tuberculosis drugs.

Table 3 depicts the univariate analysis between the patient sociodemographics and the prevalence of MDRTB. Sex (male) was the only sociodemographic factor that significantly correlated with a higher prevalence of MDR-TB (all the seven cases of MDR-TB occurred in male individuals).

\begin{tabular}{|l|l|}
\hline Sociodemographic variable & p-value \\
\hline Nationality & 0.244 \\
\hline Sex & 0.04 \\
\hline Tuberculosis type & 0.726 \\
\hline Diabetes mellitus & 0.541 \\
\hline Renal failure & 0.681 \\
\hline Acquired immunodeficiency syndrome & 0.641 \\
\hline Immunosuppression & 0.710 \\
\hline Cancer & 0.744 \\
\hline Bacillus Calmette-Guérin vaccine & 0.511 \\
\hline Previous history of tuberculosis & 0.511 \\
\hline
\end{tabular}

TABLE 3: Univariate analysis (two-tailed chi-squared test) between patient sociodemographics and prevalence of multidrug-resistant tuberculosis.

Statistical significance was determined as $\mathrm{p}$-value $<0.05$.

\section{Discussion}

This is one of the limited studies from the Western province of Saudi Arabia to scrutinize the prevalence and associated risk factors of MDR-TB among patients from Makkah. Our study revealed that the prevalence of monodrug-resistant TB ranged from 2.1\% to 3.4\%. Conversely, the prevalence rates for PDR-TB and MDRTB were $1.5 \%$. Additionally, diabetes and a previous history of TB were not substantial factors correlated with a higher occurrence of MDR-TB.

In our study, the distribution of TB cases was roughly divided between Saudi and non-Saudi nationals. This finding suggests that expatriates contribute substantially to the overall TB prevalence in Makkah, which harbors two large-sized religious gatherings and welcomes millions of people from all over the world.

Our data revealed that the prevalence rate of MDR-TB among TB-confirmed patients from Makkah was low (1.5\%). Our reported figure differed from two national registry data reporting MDR-TB prevalence rates of 
15.9\% [10] and 4.4\% [11]. Indeed, MDR-TB remains an ongoing encumbrance and several national reports from Saudi Arabia have revealed that MDR-TB rates vary substantially in accordance with the reporting region/city [12]. For example, it has been documented that the rate of MDR-TB was $1.4 \%$ in the Eastern provinces [12], 20.6\% in Najran [15], 2.5-6.7\% in Riyadh [16,17], 4\% in Al-Madinah Al-Munawarah [18], and $20 \%$ in Jeddah [19].

With regard to Makkah, only one study thus far has endeavored to examine the prevalence and determinants of MDR-TB [13]. This study was a single-center experience from the Al-Noor Specialist Hospital, reporting only a total of 158 patients. This study revealed that the prevalence rate of MDR-TB was 5\% [13]. Moreover, this study demonstrated that age, lung disease, and a previous history of TB were substantial factors correlated with MDR-TB [13]. Our present study is characterized by a larger sample size and a more holistic representation of Makkah population, as data were derived from the registry of the Central TB Laboratory in Makkah.

Isoniazid and streptomycin were the two most frequent monodrug-resistant anti-TB agents (3.4\%). Our data mirrored the findings of another study conducted in Makkah by Sambas et al. [13] and other studies conducted in Jeddah [19], Riyadh [20], and Dhahran [21]. Moreover, our data were consistent with a comparable pattern previously chronicled by two national registries in Saudi Arabia [10,11]. Indeed, isoniazid is the most commonly effective yet most frequently resistant anti-TB agent worldwide [22].

Sambas et al. [13] revealed that age, lung disease, and a previous history of TB were substantial factors correlated with MDR-TB among patients from Makkah. However, these observations did not occur in our study. The lack of statistically significant differences in our study between the abovementioned risk factors and hazard of MDR-TB could be ascribed to the small number of MDR-TB cases (1.5\%). The correlation between age and the hazard of MDR-TB is inconsistent across the published literature. While some studies revealed a positive relationship between advanced age and risk of MDR-TB [13,23], other studies from Asia [11] and Europe [24] found a negative correlation. On the other hand, several studies have identified no significant connection between age and risk of MDR-TB [25-27]. Earlier studies depicted a positive link between coexisting chronic diseases, such as diabetes mellitus and the risk of MDR-TB [28]. In addition, a prior history of TB has been extensively recognized as a substantial contributing factor to MDR-TB $[10,11]$. Our study revealed that male sex was positively correlated with a higher rate of MDR-TB, consistent with earlier systematic reviews [24,29].

It is critical to gauge the extent of resistance to $\mathrm{TB}$, particularly the prevalence rates of monodrug-resistant $\mathrm{TB}$ and MDR-TB. This is because resistance to anti-TB drugs is disadvantageously linked to limited therapeutic alternatives. Also, resistance to anti-TB drugs demands the utility of several costly drugs which are associated with high toxicities. Moreover, MDR-TB is unfavorably associated with higher morbidity and mortality compared to drug-susceptible TB. Lastly, MDR-TB is inconveniently correlated with higher healthcare costs $[7,30]$. Epidemiologic studies on anti-TB drug resistance are crucial to healthcare policymakers and stakeholders. Such studies permit the development of proper surveillance initiatives to ensure effective control and prevention of MDR-TB, along with training the treating physicians about the recommended initial anti-TB regimens for a particular geographic region.

Our study has several strengths. It is the second-ever study about the prevalence of MDR-TB in Makkah; however, with a larger sample size than that reported by Sambas et al. [13]. Our data also included only TBconfirmed cases extracted from the Central TB Laboratory in Makkah, highlighting a broader representation of the population of Makkah. Nevertheless, our study has some limitations. Such limitations comprise the retrospective and cross-sectional study design, which does not reliably permit instituting a temporal correlation between the exposure and outcome variables. Also, due to the small number of MDR-TB cases, it was not possible to confidently identify relationships between risk of MDR-TB and some clinical and demographic parameters.

\section{Conclusions}

This is the second-ever study from the Western province of Saudi Arabia to analyze the prevalence and associated risk factors of MDR-TB among patients from Makkah. Our data demonstrated that the prevalence rates of monodrug-resistant TB and MDR-TB were low (2.1\%-3.4\% and 1.5\%, respectively). Diabetes and a previous history of TB were not substantial factors correlated with a higher occurrence of MDR-TB. Additional epidemiologic studies are required to validate our results.

\section{Additional Information \\ Disclosures}

Human subjects: Consent was obtained or waived by all participants in this study. Institutional Review Board at King Abdulaziz City for Science and Technology, Riyadh, Saudi Arabia. issued approval H-01-R-012. The study protocol was approved by the Institutional Review Board at King Abdulaziz City for Science and Technology, Riyadh, Saudi Arabia. Animal subjects: All authors have confirmed that this study did not involve animal subjects or tissue. Conflicts of interest: In compliance with the ICMJE uniform disclosure form, all authors declare the following: Payment/services info: All authors have declared that no financial support was received from any organization for the submitted work. Financial relationships: All authors have declared that they have no financial relationships at present or within the previous three years with any organizations that might have an interest in the submitted work. Other relationships: All authors have declared that there are no other relationships or activities that could appear to have influenced the submitted work. 


\section{Acknowledgements}

The authors would like to thank the Ministry of Health in Saudi Arabia and the National Tuberculosis \& Prevention Program for providing the data requested. Additionally, the authors would like to thank Jomanh Al-Hayani for her help in data cleansing and preparation for analysis.

\section{References}

1. MacNeil A, Glaziou P, Sismanidis C, Date A, Maloney S, Floyd K: Global epidemiology of tuberculosis and progress toward meeting global targets - worldwide, 2018. MMWR Morb Mortal Wkly Rep. 2020, 69:281-5. 10.15585/mmwr.mm6911a2

2. Global Tuberculosis Report 2019. (2019). Accessed: April 29, 2021: https://www.who.int/teams/globaltuberculosis-programme/tb-reports/global-report-2019.

3. WHO End TB Strategy. (2015). Accessed: April 29, 2021: https://www.who.int/tb/post2015_strategy/en/\#: :text=The\%20strategy\%20aims\%20to\%20end,2020\%2C\%202025\%2C\%20and\%:

4. Villarino ME, Geiter LJ, Simone PM: The multidrug-resistant tuberculosis challenge to public health efforts to control tuberculosis. Public Health Rep. 1992, 107:616-25.

5. Khawbung JL, Nath D, Chakraborty S: Drug resistant tuberculosis: a review. Comp Immunol Microbiol Infect Dis. 2021, 74:101574. 10.1016/j.cimid.2020.101574

6. Mukherjee JS, Rich ML, Socci AR, et al.: Programmes and principles in treatment of multidrug-resistant tuberculosis. Lancet. 2004, 363:474-81. 10.1016/S0140-6736(04)15496-2

7. Rajbhandary SS, Marks SM, Bock NN: Costs of patients hospitalized for multidrug-resistant tuberculosis . Int J Tuberc Lung Dis. 2004, 8:1012-6.

8. Alene KA, Yi H, Viney K, et al.: Treatment outcomes of patients with multidrug-resistant and extensively drug resistant tuberculosis in Hunan Province, China. BMC Infect Dis. 2017, 17:573. 10.1186/s12879-0172662-8

9. Chung-Delgado K, Guillen-Bravo S, Revilla-Montag A, Bernabe-Ortiz A: Mortality among MDR-TB cases: comparison with drug-susceptible tuberculosis and associated factors. PLoS One. 2015, 10:e0119332. 10.1371/journal.pone.0119332

10. Al-Hajoj S, Varghese B, Shoukri MM, et al.: Epidemiology of antituberculosis drug resistance in Saudi Arabia: findings of the first national survey. Antimicrob Agents Chemother. 2013, 57:2161-6. 10.1128/AAC.02403-12

11. Al Ammari M, Al Turaiki A, Al Essa M, Kashkary AM, Eltigani SA, Ahmed AE: Drug resistant tuberculosis in Saudi Arabia: an analysis of surveillance data 2014-2015. Antimicrob Resist Infect Control. 2018, 7:12. 10.1186/s13756-018-0306-4

12. Ali Chaudhry L, Rambhala N, Al-Shammri AS, Al-Tawfiq JA: Patterns of antituberculous drug resistance in Eastern Saudi Arabia: a 7-year surveillance study from 1/2003 to 6/2010. J Epidemiol Glob Health. 2012, 2:57-60. 10.1016/j.jegh.2011.10.001

13. Sambas MF, Rabbani U, Al-Gethamy MM, et al.: Prevalence and determinants of multidrug-resistant tuberculosis in Makkah, Saudi Arabia. Infect Drug Resist. 2020, 13:4031-8. 10.2147/IDR.S277477

14. Tenover FC, Crawford JT, Huebner RE, Geiter LJ, Horsburgh CR Jr, Good RC: The resurgence of tuberculosis: is your laboratory ready?. J Clin Microbiol. 1993, 31:767-70. 10.1128/jcm.31.4.767-770.1993

15. Asaad AM, Alqahtani JM: Primary anti-tuberculous drugs resistance of pulmonary tuberculosis in Southwestern Saudi Arabia. J Infect Public Health. 2012, 5:281-5. 10.1016/j.jiph.2012.03.005

16. Kordy FN, Al-Thawadi S, Alrajhi AA: Drug resistance patterns of Mycobacterium tuberculosis in Riyadh, Saudi Arabia. Int J Tuberc Lung Dis. 2004, 8:1007-11.

17. Alateah SM, Othman MW, Ahmed M, Al Amro MS, Al Sherbini N, Ajlan HH: A retrospective study of tuberculosis prevalence amongst patients attending a tertiary hospital in Riyadh, Saudi Arabia. J Clin Tuberc Other Mycobact Dis. 2020, 21:100185. 10.1016/j.jctube.2020.100185

18. Elhassan MM, Hemeg HA, Elmekki MA, Turkistani KA, Abdul-Aziz AA: Burden of Multidrug Resistant Mycobacterium tuberculosis Among New Cases in Al-Madinah Al-Monawarah, Saudi Arabia. Infect Disord Drug Targets. 2017, 17:14-23. 10.2174/1871526517666161116104242

19. Khan MY, Kinsara AJ, Osoba AO, Wali S, Samman Y, Memish Z: Increasing resistance of M. tuberculosis to anti-TB drugs in Saudi Arabia. Int J Antimicrob Agents. 2001, 17:415-8. 10.1016/s0924-8579(01)00298-9

20. Al-Awaidy ST, Al-Hamdan N: Drug-susceptibility pattern of mycobacterium tuberculosis among pulmonary tuberculosis patients in Riyadh, Saudi Arabia. J Family Community Med. 1997, 4:65-9.

21. Al-Tawfiq JA, Al-Muraikhy AA, Abed MS: Susceptibility pattern and epidemiology of Mycobacterium tuberculosis in a Saudi Arabian hospital: a 15-year study from 1989 to 2003. Chest. 2005, 128:3229-32. 10.1378/chest.128.5.3229

22. Sulis G, Pai M: Isoniazid-resistant tuberculosis: a problem we can no longer ignore. PLoS Med. 2020, 17:e1003023. 10.1371/journal.pmed.1003023

23. Mehari K, Asmelash T, Hailekiros H, Wubayehu T, Godefay H, Araya T, Saravanan M: Prevalence and Factors Associated with Multidrug-Resistant Tuberculosis (MDR-TB) among Presumptive MDR-TB Patients in Tigray Region, Northern Ethiopia. Can J Infect Dis Med Microbiol. 2019, 2019:2923549. $10.1155 / 2019 / 2923549$

24. Faustini A, Hall AJ, Perucci CA: Risk factors for multidrug resistant tuberculosis in Europe: a systematic review. Thorax. 2006, 61:158-63. 10.1136/thx.2005.045963

25. Demile B, Zenebu A, Shewaye H, Xia S, Guadie A: Risk factors associated with multidrug-resistant tuberculosis (MDR-TB) in a tertiary armed force referral and teaching hospital, Ethiopia. BMC Infect Dis. 2018, 18:249. 10.1186/s12879-018-3167-9

26. Metanat M, Sharifi-Mood B, Shahreki S, Dawoudi SH: Prevalence of multidrug-resistant and extensively drug-resistant tuberculosis in patients with pulmonary tuberculosis in Zahedan, Southeastern Iran. Iran Red Crescent Med J. 2012, 14:53-5.

27. Mekonnen F, Tessema B, Moges F, Gelaw A, Eshetie S, Kumera G: Multidrug resistant tuberculosis: prevalence and risk factors in districts of metema and west armachiho, Northwest Ethiopia. BMC Infect Dis. 2015, 15:461. 10.1186/s12879-015-1202-7

28. Tegegne BS, Habtewold TD, Mengesha MM, Burgerhof JG: Association between diabetes mellitus and multidrug-resistant tuberculosis: a protocol for a systematic review and meta-analysis. Syst Rev. 2017, 6:6. 10.1186/s13643-017-0407-9

29. Pradipta IS, Forsman LD, Bruchfeld J, Hak E, Alffenaar JW: Risk factors of multidrug-resistant tuberculosis: 


\section{Cureus}

a global systematic review and meta-analysis. J Infect. 2018, 77:469-78. 10.1016/i.jinf.2018.10.004

30. Laurence YV, Griffiths UK, Vassall A: Costs to health services and the patient of treating tuberculosis: a systematic literature review. Pharmacoeconomics. 2015, 33:939-55. 10.1007/s40273-015-0279-6 